\title{
Piercing a Liquid Droplet by a Gas Jet
}

\author{
Maryam Ebrahimiazar, Amirreza Amighi, Nasser Ashgriz \\ Department of Mechanical and Industrial Engineering \\ University of Toronto \\ Toronto, Ontario, Canada
}

\begin{abstract}
Impingement of a gas jet on a suspended water droplet is studied experimentally. The jet penetration process depends on the jet velocity and jet to droplet diameter ratio. At small jet to diameter ratios and large enough jet velocities, the jet penetrated through the droplet. At larger jet to droplet diameter, the droplet fragmentation becomes more significant, with large droplet deformation. For this experiment, different types of breakup process are categorized, and theoretical explanations are provided.
\end{abstract} bility

Keywords-droplet breakup, atomization,Rayleigh-Taylor insta-

\section{INTRODUCTION}

Fragmentation of a liquid into small droplets by a gaseous jet has numerous industrial applications. Although the fragmentation process is studied extensively, it still cannot be predicted accurately. All of the presently available models are based on empirical correlations and/or simplified physical models. For instance, the breakup of a liquid droplet is experimentally studied and the breakup process is categorized based on a Weber number:

$$
W e=\frac{\rho U^{2} D_{d r o p}}{\sigma}
$$

where $\rho$ can be the density of the gas or the liquid, $U$ is the relative velocity between the gas and droplet, $D_{\text {drop }}$ is the droplet diameter, and $\sigma$ is the surface tension. It is also found that the viscosity of the fluid that the droplet is composed of also plays a role in preventing the deformation and dissipating the energy from the aerodynamic forces imposed on the droplet. The ratio of droplet viscous forces to surface tension forces can be quantified using the Ohnesorge number [2]:

$$
O h=\frac{\mu_{l}}{\sqrt{\rho_{l} D_{\text {drop }} \sigma}}
$$

where $\mu_{l}$ and $\rho_{l}$ are the droplet viscosity and density,respectively. When the $O h$ umber is greater than 0.1 , drop viscous forces are significant and should be accounted for.Different types of droplet breakups are identified and categorized based on the Weber number: Vibrational $(0<$ We $<\sim 11)$, bag $(\sim 11<W e<\sim 35)$, multimode $(\sim 35$ $<W e<\sim 80)$, sheet thinning $(\sim 80<W e<\sim 350)$, and catastrophic $(W e>\sim 350)$.
There are a wide variety of methods to perform the fragmentation process. The most common method is by applying a large relative velocity between an external gas and the liquid, thus shearing off small drops from the liquid surface. The relative velocity can be induced by either accelerating the liquid and shooting it out of a nozzle, thus having a high velocity liquid jet with respect to the surrounding gas, or by applying a high velocity gas onto the surface of the liquid. Both these effects have been studied extensively. The atomization method that is introduced here is within the later category, and in particular, on the breakup of a liquid droplet by a high velocity gaseous flow.

\section{EXPERIMENTAL SETUP}

The experimental system comprises of a suspended droplet, a gaseous jet and an imaging system. A droplet is suspended at the tip of a capillary needle (see Fig.1). The droplet is suspended so that it is fixed in space and its dynamics can be studied. A capillary needle is used to continuously and easily replace the droplet. The capillary needle is connected to a syringe pump to provide the mass needed for the droplet. The size of the droplet is controlled by the syringe pump. The liquid is pumped to the needle until a desired droplet size is obtained.

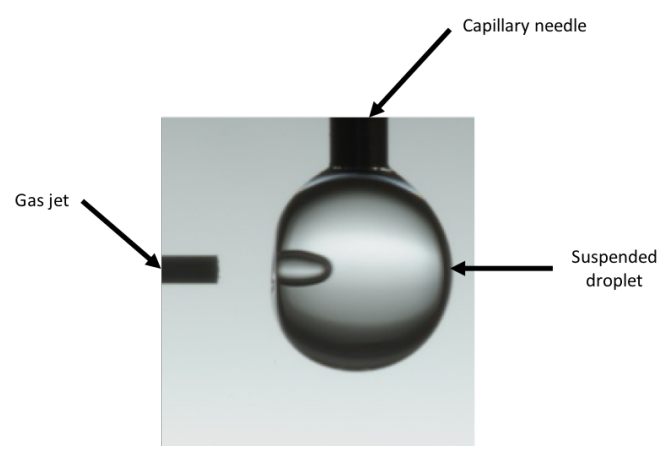

Fig. 1. Experimental Setup

A capillary needle is positioned horizontally next to the suspended droplet. The needle is connected to a highpressure air-line, through a solenoid valve. Pressure gauges are installed on the line to control the flow conditions. The 
flow is controlled by a processor. Direct visualization is used to study the droplet dynamics. Both time lapsed photography, using a still camera with a short duration flash, and a high speed video camera are used in this study. The photographs provided in this paper are from still camera. The schematic of experimental setup is shown in Fig.2.

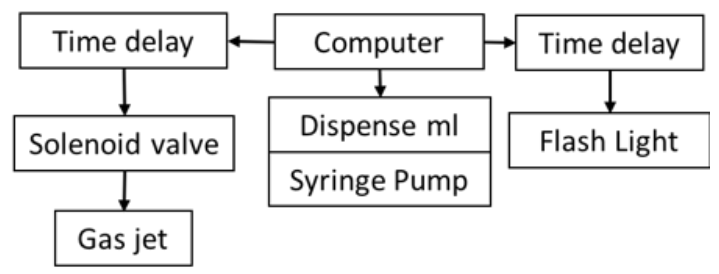

Fig. 2. Schematic of Experimental Setup

\section{Results And Discussion}

\section{A. Observations}

In our experimental study, a droplet with an average diameter of $2.6 \mathrm{~mm}$ is exposed to an air jet, and the dynamics of the droplet is studied. Droplet dynamics differ according to jet to droplet diameter ratio, as well as droplet Weber number based on air density. By progressively increasing the nozzle diameter from $0.2 \mathrm{~mm}$ to $0.8 \mathrm{~mm}$, the following dynamics were observed (Fig. 3).

1) An air tunnel is formed inside the droplet (Fig.3-1). Contrary to one's expectation, the gas jet does not just form a cylindrical tunnel inside the droplet (e.g., bullet through an apple). The gas jet slightly expands as it penetrates into the droplet. By the time the jet reaches to the end of the droplet, the front opening of the droplet is almost closed, forming a conical shape at the front side (Fig. $3-1, \mathrm{t}=0.8 \mathrm{~ms}$ ). At this time, the air jet pushes through the droplet forming another inner jet, which has the same diameter as the initial gas jet. Therefore, two different size cavities are formed inside the droplet.

2) The jet can reach to the end of the droplet and pierce through. Once the jet pierces through, another liquid front forms on the upstream side of the tunnel. In addition, capillary waves form on the drop surface. These capillary waves interact with the front opening and distort the incoming jet (Fig.3-2, $\mathrm{t}=2 \mathrm{~ms}$ ). Magnified images are shown in Fig.4.

3) In the third row, the jet reaches to the end of the droplet and then stretches the water forming a balloon (Fig.3$3, \mathrm{t}=1.2 \mathrm{~ms}$ ); the balloon burst (Fig.3-3, $\mathrm{t}=1.6 \mathrm{~ms}$ ); the balloon front retreats collapsing on itself (Fig.3-3, $\mathrm{t}=2$ $\mathrm{ms}$ ), and the incoming jet bursts it again (Fig.3-3, $\mathrm{t}=2.4$ ms). Magnified images are shown in Fig.5.

4) In this case, the jet diameter is so large that it generates a large cavity inside the droplet. Therefore, the liquid thickness around the cavity is small and susceptible to disturbances. In this case, the drop becomes very wavy in the beginning. As the balloon is formed and stretches, the liquid thickness becomes thinner. The liquid thickness around the cavity is small and susceptible to disturbances. In this case, the drop becomes very wavy in the beginning. As the balloon is formed and stretched, the liquid thickness becomes thinner and thinner and also smoother. The balloon can stretch for a long distance before it bursts. This is shown in Fig.34.

\section{B. Flow Regime Map}

In this part, the dynamics of the droplet are characterized based on the jet to droplet diameter ratio and air pressure. In regime (a), two separated cavities (bubbles) are observed in the droplet and there is a clear boundary between the two cavities (bubbles). When $\frac{D_{\text {jet }}}{D_{\text {drop }}}=0.08$ and $p=70 \mathrm{psi}$ the dynamics of the droplet starts to change. In regime (b), still two bubbles are seen in the droplet; however, there is not a clear boundary between them. In regime (c), $\frac{D_{\text {jet }}}{D_{\text {drop }}}$ is large enough to stretch the droplet and form a bag shape; meanwhile, a bubble is seen at the back of the bag shape. In regime (d), the air jet goes through the droplet, forming a large cavity inside it. The four different regimes are shown in Fig. 6.

\section{Rayleigh-Taylor Waves}

In large jet to drop diameter ratios, the air jet forms a large cavity inside the droplet, the droplet stretches, forming a front rim (windward). This liquid rim goes through RayleighTaylor (RT) instability and breaks into small droplets. The size of the droplets can be estimated based on the RT instability theory. For a RT instability, the wavelength, $\lambda_{\max }$ , corresponding to the maximum growth rate is found to be

$$
\lambda_{\text {max }}=2 \pi\left(\frac{3 \sigma}{\rho a}\right)^{1 / 2}
$$

where $a$ is the acceleration, and $\rho$ is the density of the denser fluid. To consider the effect of viscosity and surface tension, Eq.(3) changes to the following:

$$
\lambda_{\text {max }}=2 \pi\left[\left(\frac{3 \sigma}{\rho a}\right)^{1 / 2}+A_{2}\left(\frac{\mu^{2}}{\rho^{2} a}\right)^{1 / 2}\right]
$$

where $\mu$ is the dynamic viscosity of the denser fluid, and $A_{2}$ is a coefficient. This simplified equation was obtained by Aliseda et al[1], which agrees favorably with the results of their experiments. For small $O h$, the viscosity effects can be neglected, and Eq. (3) can be used instead of Eq. (4). In the present study, for large enough jet to droplet diameter ratios and large enough jet velocities, Rayleigh-Taylor waves appear at the front rim of the droplet (Fig. 7). In our case ( $O h=2.2 \times 10^{-3}$ ), Ohnesorge number is small enough to ignore the viscosity effects. Thus, we will use Eq.(3) to find the critical wavelength of Rayleigh-Taylor Instability. In Eq. (3), all the parameters are known except for the acceleration. Drop acceleration is found using a force balance[3]. The drag force is found through:

$$
F_{\text {drag }}=\frac{1}{2} \rho_{g} u^{2} C_{D} A
$$




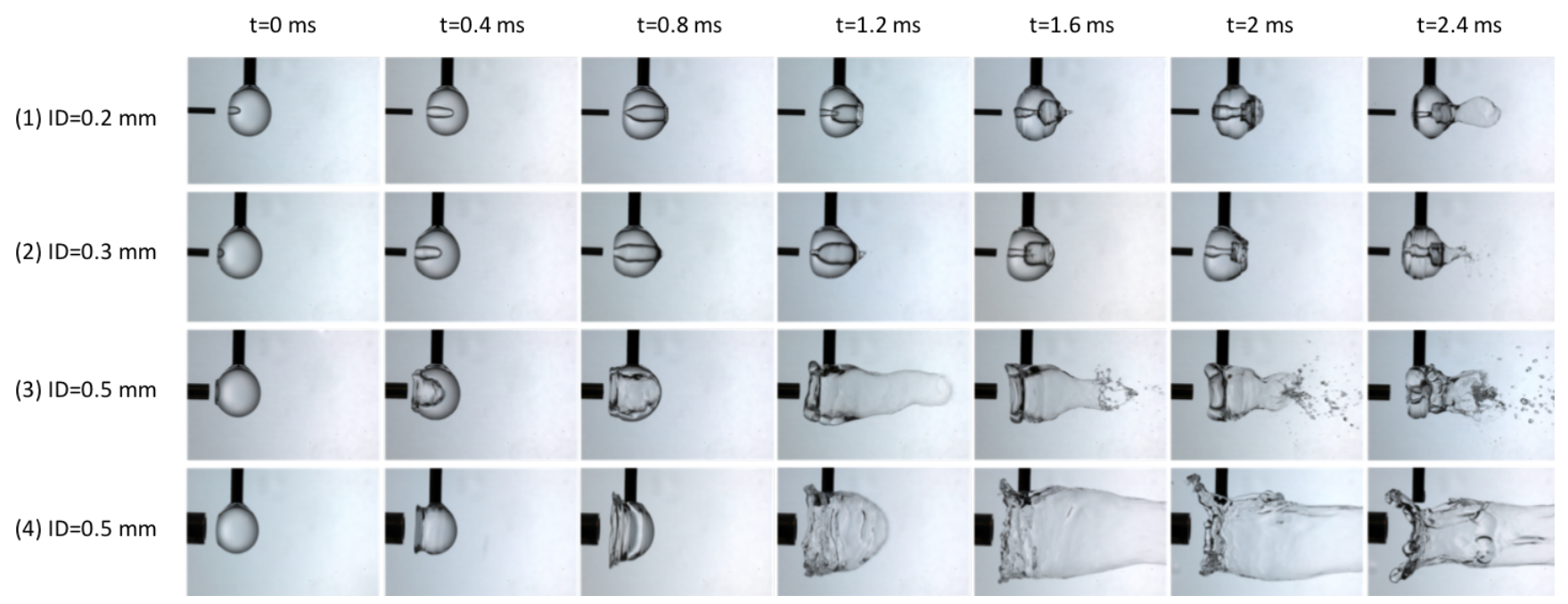

Fig. 3. Droplet dynamics for different nozzle diameters at $\mathrm{p}=10 \mathrm{psi}$

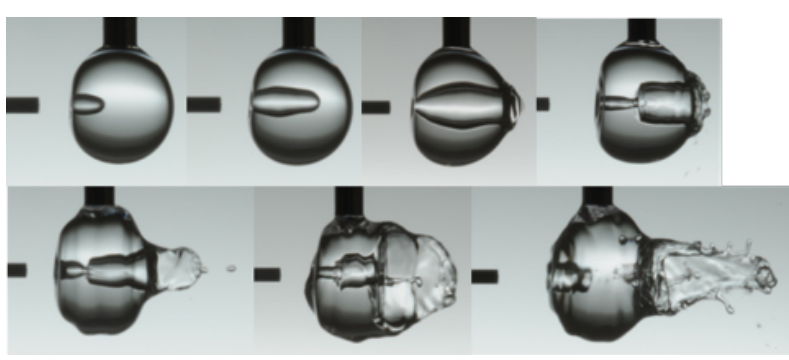

Fig. 4. Droplet dynamics for nozzle with diameter of $0.3 \mathrm{~mm}$

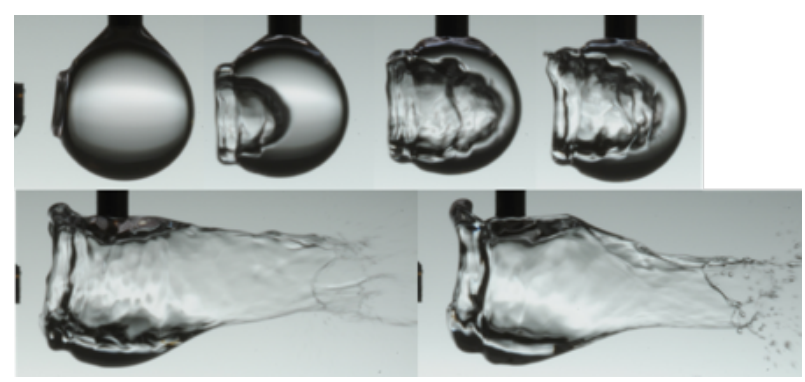

Fig. 5. Droplet dynamics for nozzle with diameter of $0.5 \mathrm{~m}$

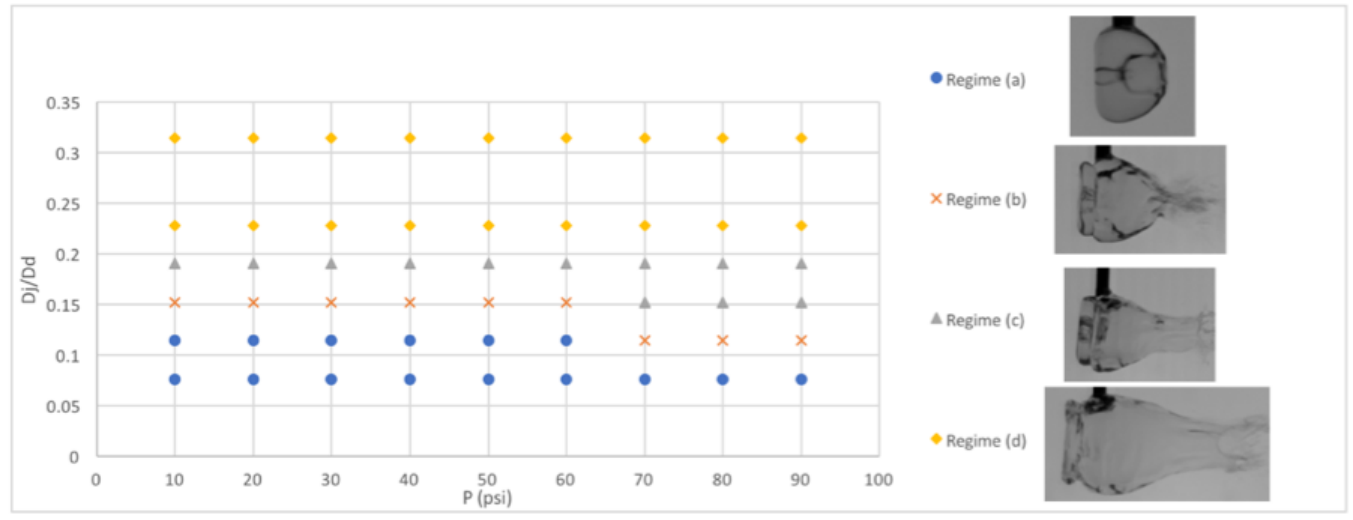

Fig. 6. Flow Regime Map

where $\rho_{g}$ is the gas density, $u$ is the air jet velocity, $C_{D}$ is the drag coefficient, and $A$ is the cross sectional area. This area is shown in Fig. 8. By this definition, the drag force equation will be

$$
F_{\text {drag }}=2 \rho_{g} u^{2} C_{D} \pi\left(R^{2}+r R\right)
$$

The other force acting on the droplet is surface tension, which can be found from Eq. (7)

$$
F=\frac{2 \sigma}{R} \times A
$$

where $\frac{1}{R}$ is the surface curvature, and $A$ is the surface on which the surface tension force is acting. Thus, the surface tension force can be written as:

$$
F=8 \pi \sigma(R+r)
$$




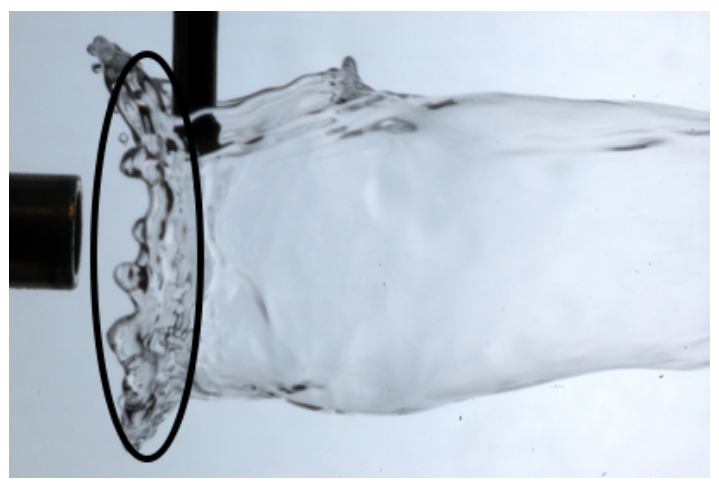

Fig. 7. Rayleigh-Taylor Waves for nozzle with diameter of $1.6 \mathrm{~mm}$ $\mathrm{L}$

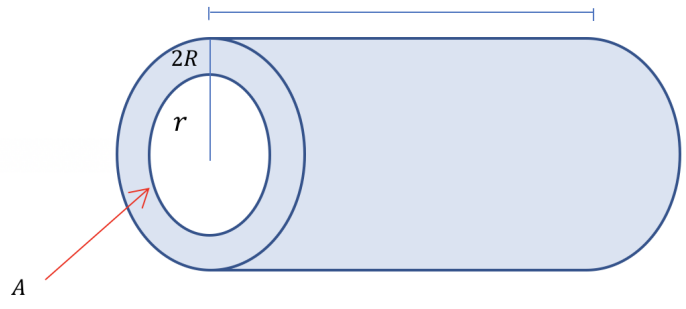

Fig. 8. Hollow cylinder forming after the jet injection

The front rim of the droplet is assumed to have a torus shape with inlet radius of $r$ and outlet radius of $R$. The mass of this torus can be found from Eq. (9).

$$
m=\frac{1}{4} \pi^{2}(2 r+2 R)(2 R)^{2}
$$

Then, the drop acceleration can be written as:

$$
a=\frac{\rho_{g} u^{2} C_{D} R+4 \sigma}{\pi R^{2} \rho_{l}}
$$

In Eq. (10), all the parameters are defined except $R$. In order to find the value of $R$, mass balance is written. The mass of the initial droplet is equal to the mass of the hollow cylinder which is formed after the air jet injection. This is shown in Eq. (11) .

$$
\pi \frac{D_{\text {drop }}^{3}}{6}=4 \pi\left(R^{2}+r R\right) L \rightarrow R=\frac{-r+\sqrt{r^{2}+\frac{D_{\text {drop }}^{3}}{6 L}}}{2}
$$

RT instabilities are observed for the $1.6 \mathrm{~mm}$ jet diameter case. For smaller nozzle diameters the front rim is smooth. The calculations are done for nozzle with diameter of 1.6 $\mathrm{mm}$, and the results are shown in Fig. 9. As the figure shows, the experimental data compare favorably with the theoretical study.

\section{Penetration Velocity}

Penetration velocity is defined as the velocity of the air jet when penetrating through the droplet from one side to the other side. By processing the images, and measuring the distance traveled by the air jet, the penetration velocity is

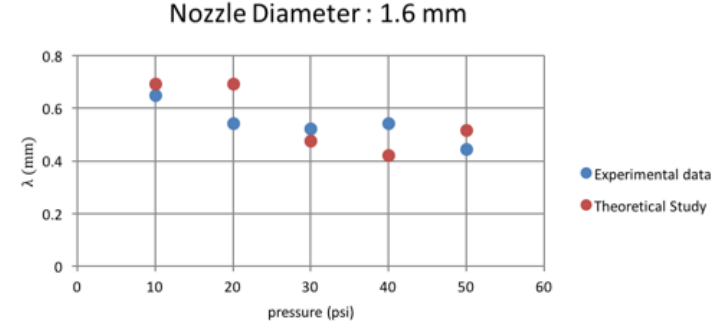

Fig. 9. Experimental and theoretical results for Rayleigh-Taylor waves

calculated. The results are shown in Fig. 10. As the figures show, the air jet is penetrating through the droplet with constant velocity. Moreover, as it is expected, by increasing the air pressure, the penetration velocity is increasing as well.

By writing a pressure balance, the penetration velocity can be found theoretically.

$$
\frac{1}{2} \rho_{a} V_{j}^{2}=\frac{1}{2} \rho_{V_{l}}^{2}+\frac{4 \sigma}{D_{d r o p}}+\frac{4 \sigma}{D_{j}}
$$

where $\rho_{a}$ and $\rho_{j}$ is air and water density, $V_{j}$ is jet velocity, $\sigma$ is the surface tension of water, $D_{d r o p}$ and $D_{j}$ are droplet and jet diameter, respectively. For nozzle with diameter of 0.2 $\mathrm{mm}$, the penetration velocity is calculated theoretically.Table 1 shows both theoretical and experimental results. As the table shows, for low pressure, the theoretical and experimental velocity are in good agreement. However, by increasing the pressure, the theoretical penetration velocity is no longer a good estimate.

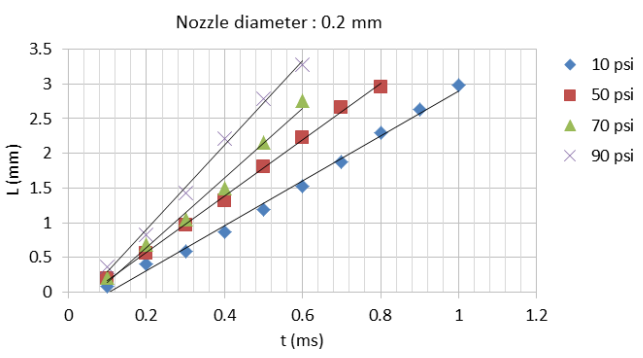

Fig. 10. Experimental penetration velocity

TABLE I

Experimental and theoretical values of penetration velocity for nozzle with diameter of $0.2 \mathrm{~mm}$

\begin{tabular}{ccc}
\hline $\begin{array}{c}\text { Nozzle Pressure } \\
(\mathrm{psi})\end{array}$ & $\begin{array}{c}\text { Experimental } \\
\text { Penetration } \\
\text { Velocity }(\mathrm{m} / \mathrm{s})\end{array}$ & $\begin{array}{c}\text { Theoretical Penetration } \\
\text { Velocity }(\mathrm{m} / \mathrm{s})\end{array}$ \\
\hline 10 & 3.24 & 3.32 \\
50 & 4.06 & 6.34 \\
70 & 5.04 & 7.52 \\
\hline
\end{tabular}

\section{REFERENCES}

[1] A Aliseda, EJ Hopfinger, Juan C Lasheras, DM Kremer, A Berchielli, and EK Connolly. Atomization of viscous and non-newtonian liquids by a coaxial, high-speed gas jet. experiments and droplet size modeling. International Journal of Multiphase Flow, 34(2):161-175, 2008. 
[2] Geoffrey Taylor. The instability of liquid surfaces when accelerated in a direction perpendicular to their planes. i. Proceedings of the Royal Society of London. Series A. Mathematical and Physical Sciences, 201(1065):192-196, 1950.

[3] Hui Zhao, Hai-Feng Liu, Xian-Kui Cao, Wei-Feng Li, and Jian-Liang Xu. Breakup characteristics of liquid drops in bag regime by a continuous and uniform air jet flow. International Journal of Multiphase Flow, 37(5):530-534, 2011. 